\title{
Effect of human seminal fluid on the growth of endometrial cells of women with endometriosis
}

\author{
Khaleque N Khan $^{\mathrm{a}^{*}}$, MD, PhD; Michio Kitajima ${ }^{\mathrm{a}}$, MD; Koichi Hiraki ${ }^{\mathrm{a}}$, MD;

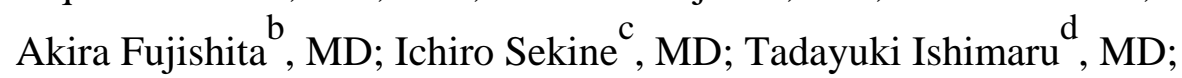 \\ Hideaki Masuzaki ${ }^{\mathrm{a}}$, MD
}

a Department of Obstetrics and Gynecology, Graduate School of Biomedical Sciences, Nagasaki University; ${ }^{b}$ Nagasaki Saiseikai Hospital, Nagasaki; ${ }^{c}$ Department of Molecular Pathology, Atomic Bomb Disease Institute, Nagasaki University, Nagasaki; ${ }^{\mathrm{d}}$ Sasebo Chuo Hospital, Nagasaki, Japan.

Running title: Effect of seminal fluid on endometriosis

*Address for correspondence and reprint request:

Khaleque Newaz Khan, MD, PhD

Department of Obstetrics and Gynecology

Graduate School of Biomedical Sciences, Nagasaki University

1-7-1 Sakamoto, Nagasaki 852-8501, Japan.

Tel: +81-95-819-7363

Fax: +81-95-819-7365

E-mail: nemokhan@nagasaki-u.ac.jp 


\section{Condensation}

Human seminal fluid enriched with different macromolecules may promote the growth of endometrial cells derived from women with endometriosis. 
Title: Effect of human seminal fluid on the growth of endometrial cells of women with endometriosis

KN Khan, M Kitajima, K Hiraki, A Fujishita, I Sekine, T Ishimaru, H Masuzaki

\begin{abstract}
Objectives: We investigated the effect of human seminal fluid on the growth of endometrial cells derived from women with and without endometriosis.
\end{abstract}

Study design: Seminal plasma (SP) was collected from 18 healthy fertile men. Sera, peritoneal fluid (PF) and tissue specimens from the eutopic and ectopic endometria were collected from 45 women with endometriosis and 20 women without endometriosis during laparoscopic surgery. Prostaglandin (PG) E2, hepatocyte growth factor (HGF), and estradiol (E2) levels in each sample of SP, sera and PF were measured by enzyme-linked immunosorbent assay. Growth pattern of cells derived from eutopic and ectopic endometria in response to SP was examined by 5-bromo-2-deoxyuridine (BrdU) incorporation assay.

Results: Seminal plasma was able to significantly stimulate the growth of epithelial cells and stromal cells derived from the eutopic and ectopic endmetria of women with endometriosis (2-3 fold) when compared with control media. The SP-promoted proliferation of both glands cells and stromal cells derived from eutopic endometria was 
also remarkably higher in women with endometriosis than that of women without endometriosis. Although levels of PGE2, HGF and E2 in SP were variable when compared with other body fluids, the levels of PGE2 and HGF in SP were significantly higher than that in either peritoneal fluid or serum of women with or without endometriosis. Pretreatment of cells with individual anti-PGE2 antibody, anti-HGF antibody and two selective estrogen receptor modulators, tamoxifen and raloxifene was unable to suppress SP-mediated growth of endometrial cells. However, pretreatment of cells with combined anti-PGE2 antibody plus anti-HGF antibody or combined anti-PGE2 antibody plus anti-HGF antibody plus tamoxifen or raloxifene was able to significantly suppress SP-promoted growth of eutopic and ectopic endometrial cells.

Conclusion: Human seminal fluid enriched with different macromolecules may promote the growth of endometrial cells derived from women with endometriosis. Our findings may hint some detrimental effect of unprotected sexual intercourse in women with endometriosis.

Key Words: Seminal plasma; macromolecules; peritoneal fluid; cell growth; endometriosis. 


\section{Introduction}

Endometriosis, the presence of functional endometrium outside of the uterine

cavity, causes abdominal pain, dysmenorrhea, dyspareunia and infertility in about $10 \%$ of the female population (1). However, an in-depth understanding of the physiopathology of endometriosis is still elusive. A number of literatures have demonstrated the potential role of ovarian steroid hormones in the regeneration of endometrium after menstruation and the growth of endometriosis (2, 3).

Endometriosis induces a variable amount of inflammatory reaction in pelvic environment depending on the staging and morphologic appearance of disease $(4,5)$. The inflammatory reaction associated with endometriosis was demonstrated both in vitro and in vivo by the infiltration of immune cells, presence of a number of primary and secondary inflammatory mediators in tissue and body fluids $(6,7)$. With regard to etiology, genetic factors may also be involved in the development of endometriosis $(8,9)$. It appears that all the triggering factors in the generation or in the regulation of this enigmatic disease reside in an in vivo environment of female reproductive tract either in intrauterine cavity, ovary or in pelvic cavity.

While endogenous factors were enormously reported, different exogenous 
factors for the regulation of endometriosis are scarcely described. Some endocrine disruptors and bacterial or viral infection were found to be involved in the regulation or malignant transformation of endometriosis $(7,10-12)$. Since, human seminal fluid is enriched with different macromolecules, information regarding the effect of seminal fluid, as an additional exogenous factor, on the changes in endometrial cell growth is unknown. Seminal fluid of bovine animals and humans contains a number of macromolecules, such as cytokines, chemokines, growth factors, steroid hormones, and immune cells $(13,14)$. The entry of seminal fluid-enriched macromolecules into endometrium and pelvic cavity as a consequence of hematogenous dissemination after tissue absorption and local tissue perfusion has been described $(15,16)$. Therefore, there is a possibility that some macromolecules of seminal fluid can approach either to endometrial bed or to endometriotic lesions after sexual intercourse and may exhibit some biological changes in these tissues. Here we report that human seminal fluid retains some active macromolecules that may promote the growth of endometrial cells derived from women with and without endometriosis.

\section{Material and Methods}

Collection of seminal plasma. Ejaculates from 18 healthy and fertile male 
donors with normal spermiogram according to the WHO criteria (17) were collected and liquefied. Seminal plasma (SP) was extracted by centrifugation at $700 \mathrm{~g}$ for $10 \mathrm{~min}$. A second round of centrifugation was performed at 10,000 g for $10 \mathrm{~min}$. The supernatant, SP, from each donor was collected and stored at $-20^{\circ} \mathrm{C}$ until subsequent bioassay. An aliquot of fresh (non-frozen) SP was used for experiments in primary culture.

Collection of PF and serum. Peritoneal fluid (PF) and sera were collected from 45 women with endometriosis and 20 women without endometriosis during and before laparoscopy, respectively. Women with endometriosis between 18 and 44 years of age underwent laparoscopy for pelvic pain, dysmenorrhoea, and/or infertility and endometriosis free women between 17-39 years of age were operated for dermoid cyst or other benign ovarian tumors by laparoscopy. The staging and the morphological distribution of peritoneal lesions were based on the revised classification of the American Society of Reproductive Medicine (r-ASRM, 1997) (18). Biopsy specimens were collected during laparoscopy and the diagnosis of endometriosis was confirmed in all women by histopathology. Neither the study group nor the endometriosis free group had been on hormonal medication in the 3 months prior to the surgical procedure. All control 
women and women with endometriosis had regular menstrual cycles (28-32 days). The details of physical collection and preparation of PF and sera were reported elsewhere (5, 6). All samples of PF and sera were stored at $-70^{\circ} \mathrm{C}$ until assayed. All tissues and fluid samples were collected in accordance with the guidelines of the Declaration of Helsinki and with the approval by the Nagasaki University Institutional Review Board. An informed consent was obtained from all male volunteers and women.

Isolation of cells in primary culture. Stromal cells and epithelial cells were collected from the biopsy specimens of the eutopic and ectopic endometria derived from 30 women with endometriosis and 20 women without endometriosis during laparoscopy. The detail procedure of the isolation of cells in primary culture is described previously $(19,20)$. All these cells derived from individual women were subsequently used for their growth potential in response to SP.

The characteristics of the cultured stromal cells and epithelial cells were determined by morphological and immunocytochemical studies. The isolated cells were placed in four-chamber slide (Nunc, Naperville, IL). After 24 hours, the slides were washed in PBS, fixed with 4\% paraformaldehyde for 10 minutes, and rinsed with PBS. 
Slides then were incubated in $0.1 \%$ Triton X-100 for 5 minutes and incubated for 3 hour in $37{ }^{\circ} \mathrm{C}$ as follows: against human cytokeratin monoclonal antibodies (mAb) (epithelial-cell specific) at a dilution of 1:50 (MNF 116;Dako, Denmark), against human vimentin mAb (stromal cell specific) at a dilution of 1:20 (V9; Dako), against human von Willebrand factor mAb (endothelial-cell specific) at a dilution of 1:50 (Dako), and against CD45 mAb (other leukocytes) at a 1:50 (Dako) dilution. The specificity of the immunocytochemical staining was confirmed by immunoreaction to non-immune mouse immunoglobulin (Ig) G1 antibody at a 1:50 (Dako) dilution. The purity of stromal cell and epithelial cell preparation was more than 95\%, as judged by positive cellular staining for vimentin and cytokeratin, respectively.

Cell proliferation assays. 5-Bromo-2-deoxyuridine (BrdU) labeling and detection kit measures cell proliferation by quantitating BrdU incorporated into the newly synthesized DNA of replicating cells (21). The incorporated BrdU can be detected by a quantitative cellular enzyme immunoassay (Biotrak, Amersham Pharmacia Biotech Ltd., UK) using monoclonal antibodies directed against BrdU. It offers a non-radioactive alternative to the $\left[{ }^{3} \mathrm{H}\right]$-thymidine-based cell proliferation and carries equal sensitivity and 
specificity (21). The detail procedure of BrdU incorporation assay was described previously $(6,19,20)$. We first examined the proliferation of stromal cells and epithelial cells (initial plating, $10^{5} / \mathrm{ml} /$ well) in response to SP in serial dilution (1:100 to $1: 1000$ ) and after an incubation period of 24 hrs. We used Phenol Red-free DMEM media with 5\% charcoal-stripped FBS throughout the experiment for the initial culture of cells. In order to examine the individual and combined effect of PGE2, HGF and E2 presenting in SP on cell growth, respective cells $\left(10^{5} / \mathrm{ml} /\right.$ well) were pre-treated with anti-PGE2 antibody (10 $\mu \mathrm{g} / \mathrm{mL}$, Sigma Chemical Co. USA), anti-HGF antibody (10 $\mu \mathrm{g} / \mathrm{mL}, \mathrm{R} \& \mathrm{D}$ system, USA), and two selective estrogen receptor modulators (SERM), tamoxifen and raloxifene $\left(10^{-6} \mathrm{~mol} / \mathrm{L}\right.$ each, both from Sigma Chemical Co. USA). After 20min of incubation, these cells were treated with SP (1:200). Any change in cell proliferation was examined after a further incubation period of $24 \mathrm{hrs}$ and are expressed as the percentage of controls (only culture media-treated cells). The specificity of the inhibitory effects of the antibodies was examined by parallel treatment of cells with mouse IgG2a (20 $\mu \mathrm{g} / \mathrm{mL}$ ) adjunction in the culture medium.

PGE2, HGF and E2 bioassay in SP, PF and sera. The concentrations of PGE2 
and HGF in each sample of SP, PF and sera were measured in duplicate using a commercially available sandwich enzyme-linked immunosorbent assay (ELISA) developed by R \& D system in a blind fashion (Quantikine, R \& D system, Minneapolis, MN) and estimated the mean for all cases. The antibodies used in PGE2 and HGF determination do not cross-react with other cytokines. The levels of estradiol (E2) in each sample of SP, PF and sera were measured by a modified immulize-enzyme amplified luminescence system and as described previously (22). The detection limits were 6.78 $\mathrm{pg} / \mathrm{ml}$ for PGE2, $40.0 \mathrm{pg} / \mathrm{ml}$ for HGF, and $20.0 \mathrm{pg} / \mathrm{ml}$ for E2. Both the intra-assay and inter-assay coefficients of variation were $<10 \%$ for all these assays.

Statistical analysis. The clinical characteristics of the subjects were evaluated by one-way analysis of variance. The data were expressed as either mean \pm SEM or \pm SD or median and interquartile range (IQR). The differences between two groups were compared using non-parametric Mann-Whitney U-test and linear regression analysis. For comparison among three or more groups, the Kruskal-Wallis test was used to determine the difference among the groups. $\mathrm{P}<0.05$ was considered statistically significant.

\section{Results}

The mean ages of the control group and the women with endometriosis were 
$29.2 \pm 3.9$ years and 31.6 \pm 4.2 years $( \pm S D$ ), respectively. The distribution of the phases of menstrual cycle between these two groups of women, dominant endometriotic lesions in the peritoneal cavity and r-ASRM staging of endometriosis are shown in Table 1. There were no significant differences in ages and other clinical characteristics between women with endometriosis and without endometriosis.

SP-stimulated growth of eutopic and ectopic endometrial cells. All SP samples were individually tested for their proliferative effects on eutopic and ectopic endometrial cells and we found similar fashion of cell growth in response to each SP sample. As shown in Figure 1 (A and B), we found that direct application of SP stimulated dilution-dependent growth of epithelial cells and stromal cells derived from the eutopic endometria of women with endometriosis and without endometriosis. A similar pattern of cell growth in response to SP was also observed for cells derived from ectopic endometria of women with endometriosis (Figure 1, C). The SP-mediated growth of endometrial cells was remarkably higher (2-3 fold) when compared with SP-non-treated cells. The SP-promoted (1:100 to 1:300 dilution) growth of both epithelial cells and stromal cells derived from the eutopic endometria of women with endometriosis 
was significantly higher ( $<<0.05$ for each dilution) when compared with that of similar cells derived from women without endometriosis (Figure 1, A and B). Although a 1.7-to 3-fold and significant increase in the proliferation of epithelial cells and stromal cells derived from ectopic endometria ( $\mathrm{p}<0.001$ or $\mathrm{p}<0.05$ vs. control cells) was observed in response to 1:100-1:500 dilution of SP, this difference in cell proliferation was lost with further dilution of SP (1:750-1:1000) (Figure 1, C).

PGE2, HGF and E2 levels in different body fluids. We measured levels of PGE2, HGF and E2 in each sample of SP (1:100) of healthy volunteers, PF and sera of women with and without endometriosis. Using Kruskal-Wallis analysis, we found that among three different body fluids, levels of PGE2 and HGF in SP was significantly higher than that in PF and sera derived from women with endometriosis and non-endometriosis ( $\mathrm{p}<0.01$ for each) (Figure 2, A, B). No apparent difference in E2 levels was observed among these three different body fluids (Figure 2, C). When compared between groups, PGE2 and HGF concentrations in the PF of women with endometriosis were significantly higher than those of women without endometriosis $(\mathrm{p}<0.05$ for each, Figure 2, A, B). Although data not shown, there was no significant difference in the levels 
of either PGE2 or HGF between the proliferative phase and the secretory phase of the menstrual cycle.

Blocking experiments and changes in cell growth. In order to examine which macromolecule in SP might be responsible for the promotion of cell growth, we performed blocking experiments with respective antibodies against PGE2 and HGF and with two SERMS. Since the proliferative effect of SP on epithelial cells and stromal cells derived from the eutopic and ectopic endometria of women with endometriosis was almost the same at two different dilutions (1:100 and 1:200) of SP, we used higher dilutions of SP (1:200) for our blocking experiments. We found that individual co-culture of epithelial cells and stromal cells pre-treated with each of these antibodies or SERMS failed to suppress SP (1:200)-promoted growth of these cells (Figure 3). In contrast, combined co-culture of cells pre-treated with either double antibodies (anti-PGE2 + anti-HGF) or double antibodies + SERM (anti-PGE2 + anti-HGF + tamoxifen) were found to significantly suppress SP (1:200)-promoted growth of epithelial cells $(\mathrm{p}<0.05)$ and stromal cells $(\mathrm{p}<0.05)$ (Figure 3$)$ derived from the ectopic endometria of women with endometriosis. A similar growth suppression effect was also observed when we added 
raloxifene instead of tamoxifen with other two antibodies and when we used epithelial cells and stromal cells derived from eutopic endometria of women with and without endometriosis (data not shown). No inhibitory effect of cell proliferation was found when these cells were treated with control IgG2a adjunction in the culture medium.

\section{Discussion}

We demonstrated for the first time that direct application of seminal plasma has the capacity to stimulate proliferation of endometrial and endometriotic cells derived from women with endometriosis. This biologic effect of SP was significantly higher for endometrial cells derived from women with endometriosis when compared with that of non-endometriosis. The SP-promoted cell proliferation may be due to the combined effect of some active macromolecules in SP such as PGE2, HGF and E2.

The direct growth promoting effect of HGF and E2 on endometrial cells has been described previously $(6,19)$. This time, we reported that seminal fluid containing these macromolecules including PGE2 stimulated the growth of endometrial and endometriotic cells. Besides other macromolecules, PGE2 is also able to enhance cell proliferation, suppress cell apoptosis and induce the production of some proteolytic 
enzymes with the net result of cell survival and invasion $(23,24)$. In fact, endometriotic cells retain the similar properties. The multifunctional role of HGF in the development of endometriosis has been recently reported $(7,25)$. Basically, endometriosis is one of the reproductive diseases and is estrogen dependent $(2,7)$. For these reasons, we decided to measure PGE2, HGF and E2 in SP for our current study. Since seminal plasma is enriched with a number of macromolecules $(13,14)$, we speculated in this study that SP could be an additional trigger in the growth of cells derived from women with endometriosis.

Seminal fluid itself or different macromolecules retaining in SP can enter into endometrial bed or into peritoneal bed as a result of hematogenous dissemination or direct tissue perfusion through anterior or posterior vaginal fornix after sexual intercourse (15). According to recent literatures, seminal fluid is enriched with different macromolecules, such as immune cells, cytokines, chemokines, growth factors, and sex steroid hormones $(13,14)$. Once these macromolecules are available to endometrial or peritoneal bed, some of these macromolecules may stimulate the growth of endometrial or endometriotic cells. In fact, we found that SP containing a variable concentration of PGE2, HGF and E2 was 
able to significantly stimulate the growth of endometrial cells derived from the eutopic and ectopic endometria of women with endometriosis when compared with similar cells derived from non-endometriosis or SP-non-treated cells. This was supported by the findings that when we co-cultured endometrial cells with SP pre-treated with either double antibodies against PGE2 and HGF or double antibodies plus either of two SERMs such as tamoxifen or raloxifene, SP-promoted growth of cells was significantly suppressed. Basically, the histogenesis and proliferative potentiality are different between endometrial cells derived from women with endometriosis and control women as we reported previously $(6,7)$. This basic property of eutopic endometrial cells derived from women with endometriosis may predispose them to proliferate more in response to SP when compared with similar cells derived from control women.

Tamoxifen and raloxifene are two potential SERMs that function as estrogen receptor antagonist in some tissues, but have either partial or full agonist activity in others $(26,27)$. In contrast to the apparent estrogenic effect in the postmenopausal endometrium, an anti-estrogenic activity of tamoxifen in the presence of ovarian estrogen secretion has been suggested (28). Opposed to tamoxifen, there is ample clinical evidence that 
raloxifene does not stimulate the endometrium $(29,30)$. We found a small amount of estrogen in SP and the presence of local estrogen in SP may explain the partial anti-estrogenic activity of tamoxifen in association with other blocking reagents. Therefore, we presume that the combined effect of these blocking agents may suppress the SP-promoted growth of endometrial cells in our current study.

Recent studies demonstrated that exposure to semen directly promotes embryo development and implantation and thereby improves pregnancy rates. This effect of seminal fluid on successful pregnancy is contributed by the expansion of CD4+ CD25+ T regulatory cell pool that induces tolerance to paternal alloantigens $(31,32)$. However, the detrimental effect of SP on reproduction is elusive. We speculated that this is not due to the direct effect of SP rather its indirect effect on the growth of endometriosis as reported here. The different macromolecules, such as reactive oxygen species or tumor necrosis factor alpha as produced by the endometriotic cells themselves or by the infiltrated macrophages, are reported to have detrimental effect on reproductive outcome (33). In addition to this, inflammatory adhesion in response to endometriotic lesions may be involved in the adverse reproductive outcome (33). We need further studies to clarify the 
detrimental effect of SP on reproduction.

In conclusion, we suggest that as an additional exogenous factor, human seminal fluid enriched with some active macromolecules may be able to stimulate the growth of endometrial cells derived from women with endometriosis. Our current findings have some clinical implications. (i) Seminal fluid after unprotected sexual intercourse may worsen the growth of cells in women with coexisting endometriosis. Our findings may support some detrimental effect of unprotected sexual intercourse in women with endometriosis. (ii) In ART clinics, more strict measures are necessary to remove macromolecules of seminal fluid during intrauterine insemination for women who already harbor endometriosis. Further studies are necessary to examine the changes in the growth of endometriotic lesions after insemination in bovine animals or in humans and in order to strengthen our current findings.

Acknowledgements: We thank Miss Kazumi Hayashida and Miss Kyoko Ishida, Department of Obstetrics and Gynecology, Nagasaki University Graduate School of Biomedical Sciences, Nagasaki, Japan, for their excellent technical assistance. 
Conflict of interest: There is no conflict of interest related to this study.

Funding: This work was supported by Grants-in-Aid for Scientific Research (Grant No. 16591671 and 18591837) from the Ministry of Education, Sports, Culture, Science and Technology of Japan (to KN Khan).

\section{References}

(1) Strathy JH, Molgaard CA, and Coulman CB. Endometriosis and infertility: a laparoscopic study of endometriosis among fertile and infertile women. Fertil Steril 1982;38:667-672.

(2) Fujishita A, Nakane PK, Koji T, Masuzaki H. Chavez RO, Yamabe T, Ishimaru T. 
Expression of estrogen and progesterone in endometrium and peritoneal endometriosis: an immunohistochemical and in situ hybridization study. Fertil Steril 1997;67:856-864.

(3) Nisolle M, Casanas-Rouz F, Donnez J. Immunohistochemical analysis of proliferative activity and steroid receptor expression in peritoneal and ovarian endometriosis. Fertil Steril 1997;68:912-919.

(4) Halme J, Becker S, and Haskill S. Altered maturation and function of peritoneal macrophages: possible role in pathogenesis of endometriosis. Am J Obstet Gynecol 1987;156:783-789.

(5) Khan KN, Masuzaki H, Fujishita A, Kitajima M, Sekine I, Ishimaru T. Differential macrophage infiltration in early and advanced endometriosis and adjacent peritoneum. Fertil Steril 2004;81:652-661.

(6) Khan KN, Masuzaki H, Fujishita A, Kitajima M, Kohno T, Sekine I, Matsuyama T, Ishimaru T. Regulation of hepatocyte growth factor by basal and stimulated macrophages in women with endometriosis. Hum Reprod 2005a;20:49-60.

(7) Khan KN, Kitajima M, Hiraki H, Fujishita A, Sekine I, Ishimaru T, Masuzaki H. Immunopathogenesis of pelvic endometriosis: role of hepatocyte growth factor, 
macrophages and ovarian steroids. Am J Reprod Immunol (review), 60:383-404, 2008.

(8) Prowse AH, Manek S, Varma R, Liu J, Godwin AK, Maher ER, Tomlinson IP, Kennedy SH. Molecular genetic evidence that endometriosis is a precursor of ovarian cancer. Int J Cancer 2006;19(3):556-562.

(9) Guo SW. Association of endometriosis risk and genetic polymorphisms involving sex steroid biosynthesis and their receptors: a meta-analysis. Gynecol Obstet Invest 2006;61(2):90-105.

(10) Caserta D, Maranghi L, Mantovani A, Marci R, Maranghi F, Moscarini M. Impact of endocrine disruptor chemicals in gynecology. Hum Reprod Update 2008;14(1):59-72.

(11) Kodati VL, Govindan S, Movva S, Ponnala S, Hasan Q. Role of Shigella infection in endometriosis: a novel hypothesis. Medical Hypothesis 2008;70:239-243.

(12) Oppelt P, Renner SP, Strissel PL, Beckmann MW, Strick R. Detection of viruses in endometriosis lesions. Proceeding of the $10^{\text {th }}$ World Congress on Endometriosis, Melbourne. 2008;March:p55 (abstract). 
(13) Politch JA, Tucker L, Bowman FP, Anderson DJ. Concentrations and significance of cytokines and other immunologic factors in semen of healthy fertile men. Hum Reprod 2007;22(11):2928-2935.

(14) Akinloye O, Arowojolu AO, Shittu OB, Abbivesuku FM, Adejuwon CA, Osotimehin B. Serum and seminal plasma hormonal profiles of infertile Nigerian male. Afr J Med Sci 2006;35(4):468-473.

(15) Klemmt L, Scialli AR. The transport of chemicals in semen (review). Birth Defects Res 2005;74(2):119-131.

(16) Schuberth HJ, Taylor U, Zerbe H, Waberski D, Hunter R, Rath D. Immunological responses to semen in the female genital tract. Theriogenology 2008;70:1174-1181.

(17) WHO. WHO Laboratory Manual for the Examination of Human Semen and Sperm-Cervical Interaction. Cambridge: Cambridge University Press, $4^{\text {th }}$ Edition, 1999. (18) The American Society for Reproductive Medicine. Revised American Society for Reproductive Medicine classification of endometriosis: 1996. Fertil Steril 1997;67:817-821.

(19) Khan KN, Masuzaki H, Fujishita A, Kitajima M, Sekine I, Matsuyama T, Ishimaru T. 
Estrogen and progesterone receptor expression in macrophages and regulation of hepatocyte growth factor by ovarian steroids in women with endometriosis. Hum Reprod 2005b;20:2004-2013.

(20) Khan KN, Masuzaki H, Fujishita A, Kitajima M, Hiraki K, Sekine I, Matsuyama T, Ishimaru T. Interleukin-6- and tumor necrosis factor $\alpha$-mediated expression of hepatocyte growth factor by stromal cells and its involvement in the growth of endometriosis. Hum Reprod 2005c;20:2715-2723.

(21) Takagi S. Detection of 5-bromo-2-deoxyuridine (BrdU) incorporation with monoclonal anti-BrdU antibody after deoxyribonuclease treatment. Cytometry 1993;14:640-649.

(22) Babson AL. The DPC Cirrus IMMULITE automated immunoassay system. J Clin Immunol 1991;14:83-88.

(23) Kuno S, Ueno R, Hayaishi O, Nakashima H, Harada S and Yamamoto N. Prostaglandin E2, a seminal constituent, facilitates the replication of acquired immune deficiency syndrome virus in vitro. Proc. Natl. Acad. Sci. USA 1986;83:3487-3490.

(24) Harris SG, Padilla J, Koumas L, Ray D, Phipps RP. Prostaglandins as modulators of 
immunity. TRENDS in Immunology 2002;23(3):144-150.

(25) Khan KN, Kitajima M, Fujishita A, Ishimaru T, Sekine I, Masuzaki H. Multifunctional role of hepatocyte growth factor in the development of pelvic endometriosis (review article). WES e-journal 2008;July:13-20.

(26) Labrie F. Drug insight: breast cancer prevention and tissue-targeted hormone replacement therapy. Nat Clin Pract Endocrinol Metab 2007;3:584-93.

(27) Singh MN, Stringfellow HF, Paraskevaidis E, Martin-Hirsch PL, Martin FL. Tamoxifen: important consideration of a multi-functional compound with organ-specific properties. Cancer Treat Rev 2007;33:91-100.

(28) Mylonas I, Jeschke U, Makovitzky J, Winkler I, Richter DU, Friese K, Briese V. Immunohistochemical expression of steroid receptors and Glycodelin A in isolated proliferative human glandular cells after stimulation with tamoxifen and phytoestrogens (genistein and daidzein). Anticancer Res 2003;23:1119-126.

(29) Cohen FJ, Watts S, Shah A, Akers R, Plouffe L. Uterine effects of three-year raloxifene therapy in postmenopausal women younger than age 60. Obstet Gynecol 2000;95:104-10. 
(30) Neven P, Qail D, Marin F, Creatsas G, Depypere H, Rechberger T, Liu-Léage S, Pavo I, Schmitt H, Nickelsen T. Comparing raloxifene with continuous combined estrogen-progestin therapy in postmenopausal women: Review of Euralox 1. Maturitas 2005;52:87-101.

(31) Robertson SA, Guerin LR, Bromfield JJ, Branson KM, Ahlström AC, Care AS. Seminal fluid drives expansion of the $\mathrm{CD}^{+} \mathrm{CD}^{2} 5^{+} \mathrm{T}$ regulatory cell pool and induces tolerance to paternal alloantigens in mice. Biol Reprod 2009;80:1036-1045.

(32) Robertson SA, Sharkey DJ. The role of semen in induction of maternal immune tolerance in pregnancy. Semin Immmunol 2001;13:243-254.

(33) Khan KN, Kitajima M, Hiraki H, Fujishita A, Sekine I, Ishimaru T, Masuzaki H. Toll-like receptors in innate immunity: role of bacterial endotoxin and toll-like receptor 4 in endometrium and endometriosis. Gynecol Obstet Invest (review) 2009;68:40-52.

\section{Figure Legends}

Figure 1. Shows the direct effect of seminal plasma (1:100-1:1000) on the proliferation of epithelial cells (A, upper column) and stromal cells (B, middle column) derived from the eutopic endometria (A and $\mathbf{B}$ ) of women with endometriosis (white bar) 
and without endometriosis (black bar) and as measured by the bromodeoxyuridine (BrdU) incorporation assay. A similar SP-promoted and dilution-dependent proliferation was also observed for epithelial cells (black bar) and stromal cells (white bar) derived from the ectopic endometria of women with endometriosis (C, lower column). The results are represented as percentage of control (SP-non-treated but only media-treated cells). ${ }^{*} \mathrm{p}<0.05$ vs. non-endometriosis (A and $\mathbf{B}$ ); ${ }^{* *} \mathrm{p}<0.001$ vs. control cells and ${ }^{*} \mathrm{p}<0.05$ vs. control cells at the indicated dilution $(\mathbf{C})$. The results are expressed as mean \pm SEM of triplicate cultures of cells derived from each patient.

Figure 2. Shows concentrations of PGE2 (A), HGF (B) and E2 (C) in the seminal plasma (SP) collected from healthy fertile men and in the peritoneal fluid (PF) and sera derived from women with endometriosis and without endometriosis (control). Boxes represent the distance (interquartile range) between the first (25\%) and third (75\%) quartiles, and horizontal lines in the boxes represent medians. Kruskal-Wallis test indicated that PGE2 and HGF levels in SP were significantly higher among these three groups of body fluids $(\mathrm{p}<0.01) . \mathrm{p}<0.05$, endometriosis vs. control (A and $\mathbf{B})$. C, control women; E, endometriosis women; SP, seminal plasma. 
Figure 3. Shows the neutralizing effect of individual or combined anti-PGE2 antibody, anti-HGF antibody, and tamoxifen on SP (1:200)-promoted growth of epithelial cells (black bar) and stromal cells (white bar) derived from the ectopic endometria of women with endometriosis. The results are represented as percentage of control (SP-non-treated but only media-treated cells). Co-culture of cells pretreated with individual antibody or tamoxifen failed to suppress SP (1:200)-mediated growth of endometrial cells. However, co-culture of epithelial cells and stromal cells pretreated with either double antibodies (anti-PGE2+anti-HGF) or combined anti-PGE2+anti-HGF+tamoxifen significantly suppressed SP (1:200)-mediated growth of these cells ( $\mathrm{p}<0.05$ for each). Pretreatment of cells with control mouse IgG had no effect on the suppression of cell proliferation. The results are expressed as mean \pm SEM of triplicate cultures of cells. 
Table 1. Clinical profiles of patients with and without endometriosis

control

20

17-39

$29.2 \pm 3.9$

age in yrs $($ mean \pm SD)

r-ASRM staging: (I-II/III-IV)

dominant lesions: red/black/white

menstrual cycle: prolferative/secretory

\section{endometriosis}

45

$18-44$

$31.6 \pm 4.2$

$24 / 21$

$15 / 20 / 10$

8/12
$18 / 27$

The results are expressed as mean \pm SD. r-ASRM, revised classification of the American Society of Reproductive Medicine. 

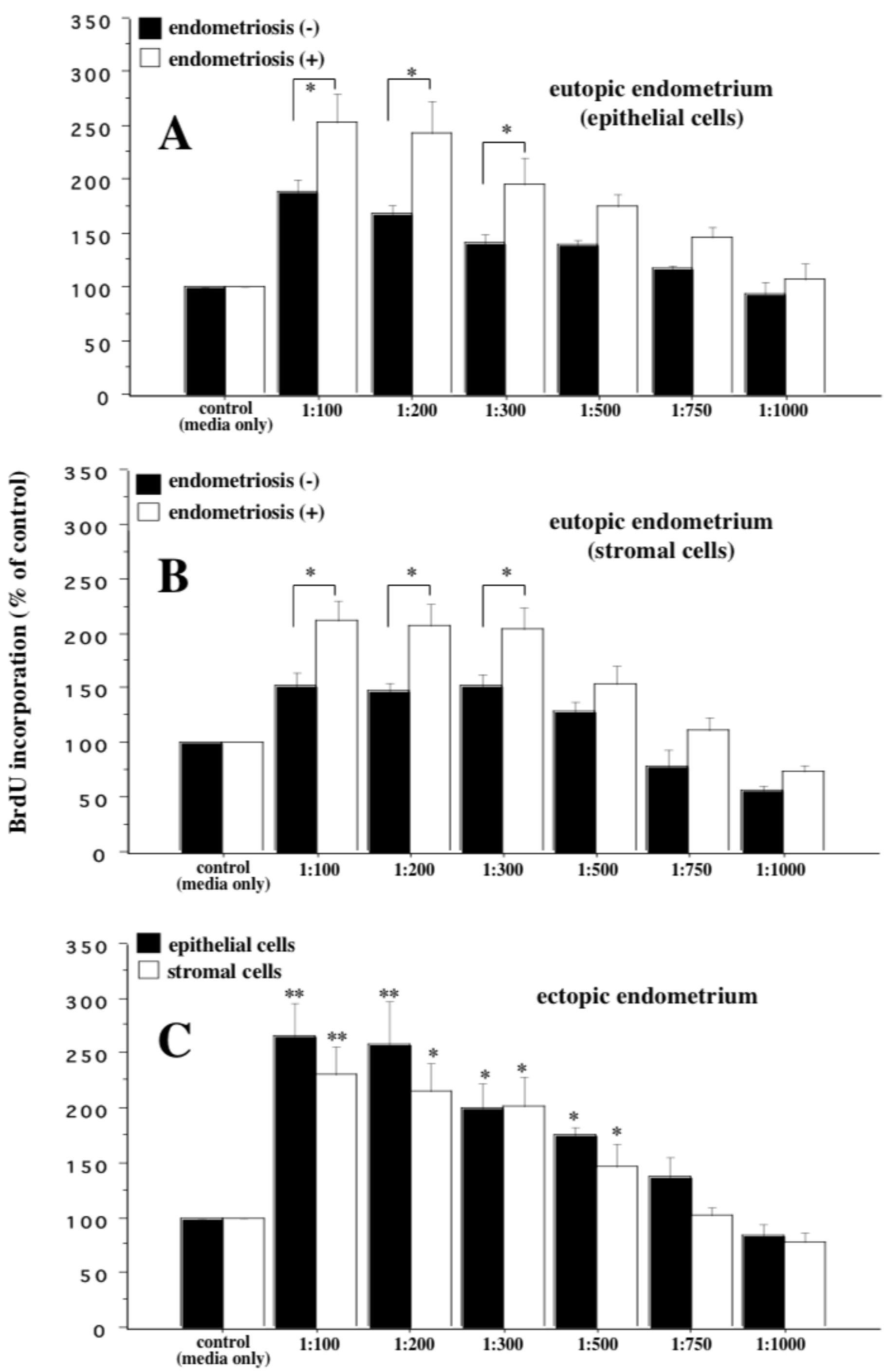

Figure 1 seminal plasma (serial dilution) 

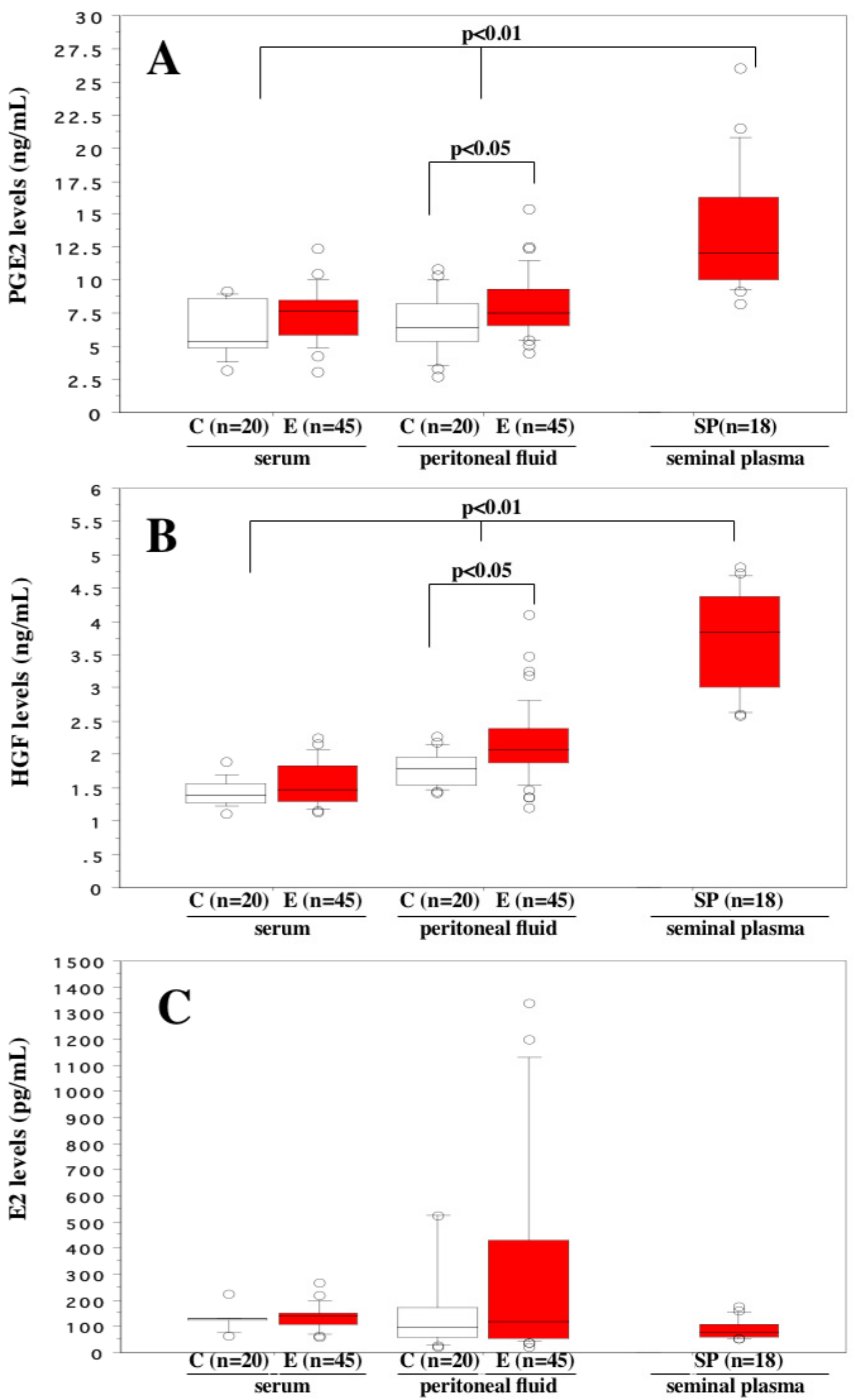

Figure 2 


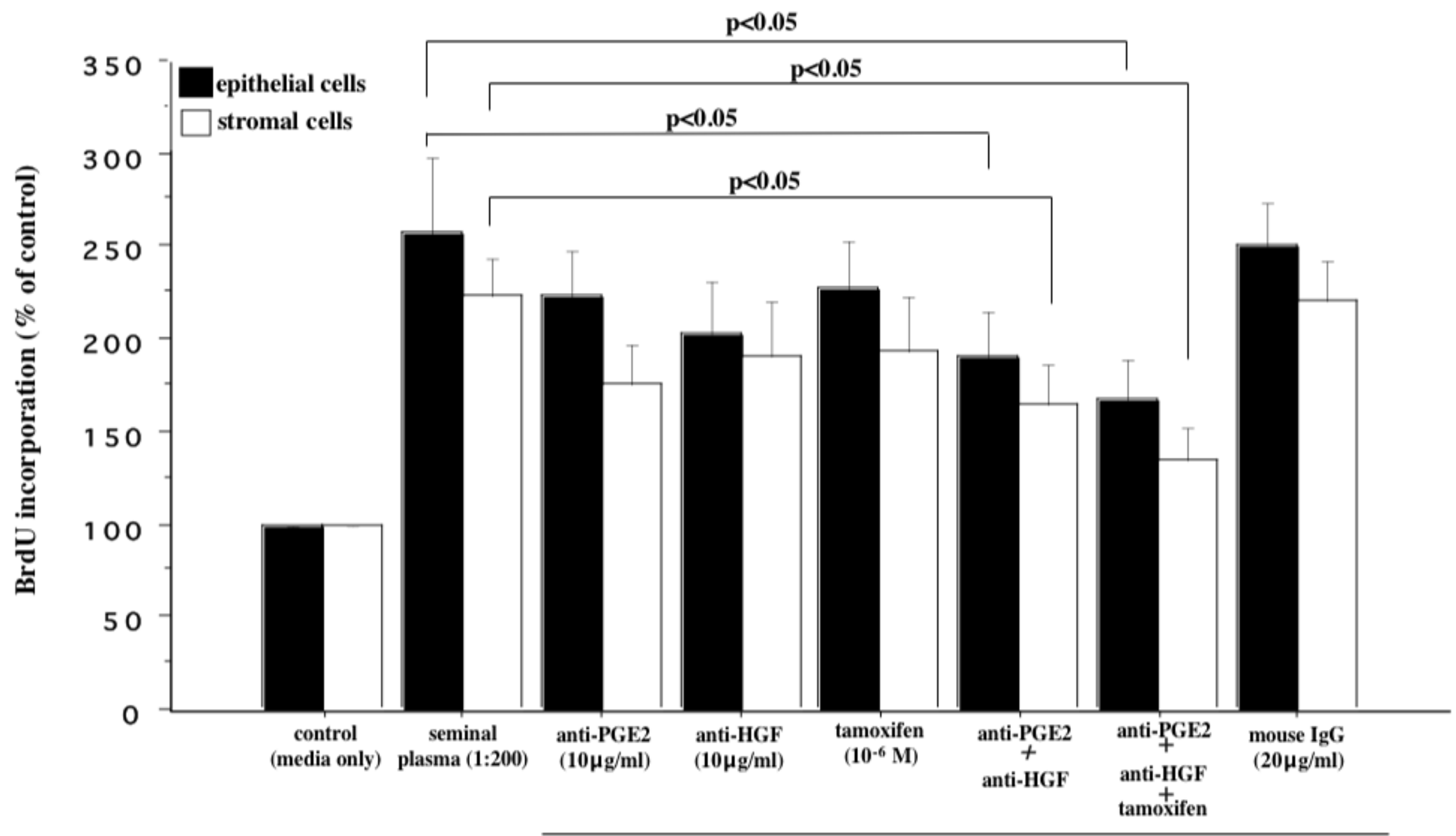

seminal plasma

Figure 3 